\title{
DSCC2010-१०००
}

\section{DYNAMIC SECURITY ANALYSIS OF ELECTRIC POWER SYSTEMS: PASSIVITY-BASED APPROACH AND POSITIVE INVARIANCE APPROACH}

\author{
Qing Hui \\ Department of Mechanical Engineering \\ Texas Tech University \\ Lubbock, TX 79409-1021 \\ Email: qing.hui@ttu.edu
}

\author{
Jinglai Shen \\ Department of Math \& Statistics \\ University of Maryland Baltimore County \\ Baltimore, MD 21250 \\ Email: shenj@umbc.edu
}

\author{
Wei Qiao \\ Department of Electrical Engineering \\ University of Nebraska-Lincoln \\ Lincoln, NE 68588-0511 \\ Email:wqiao@engr.unl.edu
}

\begin{abstract}
Security is a critical issue in modern power system operation. With the aid of analytic tools for large-scale and hybrid systems, this paper proposes two new safety verification methods for power systems. The first method is based on barrier certificates and passivity. This method provides a general safety verification framework for power systems with the port-Hamiltonian structure. The energy shaping technique is also exploited to attain safety conditions for controlled port-Hamiltonian systems. The second method, based on positive invariance, yields exact safety verification for power systems based on linearized models, particularly linear Hamiltonian systems. Decidability of exact safety verification is established via algebraic and positive invariance approaches; other analytic and numerical issues are addressed from the positive invariance perspective.
\end{abstract}

\section{INTRODUCTION}

Modern society critically relies on a securely operated electric power system to supply electricity. By nature, a power system is continually experiencing disturbances (contingencies), such as load changes, outage of generators or other equipment, short circuits, or combination of such events. These disturbances usually lead to changes in the configuration and/or state of the power system. Security refers to the degree of risk in a power system's ability to survive imminent disturbances without interruption to customer service at any instant of time [1]. In power system operation, security analysis [2], [3] is performed to determine the robustness of the system relative to imminent disturbances. For a power system subject to disturbances, it is important when the disturbances are cleared, the system settles to new operating conditions such that no physical and security con- straints are violated. This characterization of system security clearly highlights two aspects of its analysis, i.e., static security analysis and dynamic security analysis [1], [4], [5].

In static security analysis, the transition to a new operating condition is assumed to complete successfully and the analysis is aimed at verifying that no physical and security constraints are violated in the post-disturbance steady-state operating condition. However, because of the new constraints placed by environmental and economic factors, the trend in power system planning and operation is toward maximum utilization of existing infrastructure with tight system stability margins. This trend has increased the effects of disturbances on power system security. It is conceivable that, in the event of a severe disturbance, the system state may not be able to transfer quickly to a new steady-state operating point. This could trigger cascading outages in the power system and threaten system security. Therefore, dynamic security analysis is becoming more and more important in modern power system operation. In dynamic security analysis, the transition itself is of interest. This involves examining stability of the system. The majority of existing methods for dynamic security analysis fall into four categories.

Traditionally, dynamic security analysis in large disturbances, i.e., the transient stability analysis, has been performed by using nonlinear time-domain simulation [2-5]. In this method, the dynamic behavior of the generators and other components together with their interconnection through the electric power grid is modeled by a set of nonlinear differential/algebraic equations. These nonlinear equations are solved in time domain using some integration algorithm. Consequently, the dynamic behavior of the system relative to a given disturbance can be studied to determine whether stability has been maintained or lost. If instability is detected, the exact mode of instability can be 
identified. The time-domain simulation method has a few shortcomings. First, it requires intensive time-consuming numerical integration and, therefore, is not suitable for on-line applications. Second, it does not provide information regarding the degree of stability when the system is stable and the degree of instability when the system is unstable.

An alternative approach to transient stability analysis is called direct methods [5], [6-11]. The direct methods determine power system stability directly based on transient energy functions, which have some of the properties of Lyapunov functions. These methods determine whether or not the system will remain stable by comparing the system energy when the disturbance is cleared to a critical energy value. The direct methods not only avoid the time-consuming solutions of step-by-step time-domain stability analysis of the post-disturbance system, but also provide a quantitative measure of the degree of system stability and a capability to calculate sensitivities of the stability margin to power system parameters, allowing for efficient computation of operating limits. A major limitation of the direct methods is that they are impractical for large-scale power system stability analysis with detailed models. Another limitation is that the direct methods are largely limited to first swing analysis. However, in many cases transient instability may occur after the first swing if there is no sufficient damping in the system [12]. Hybrid methods [13] combining the direct methods and the time-domain simulation method have been proposed for stability analysis. However, the hybrid methods still cannot overcome the limitations of the direct methods and the time-domain simulation method.

A general safety verification of dynamical systems poses a challenging analytical and numerical problem because of infinite dimensional nature of dynamics. For this reason, two technical paths have been widely followed in the literature: one is based on approximation methods (e.g., overapproximation/under-approximation and asymptotic approximation) for general nonlinear dynamics or fast computation [14], and the other focuses on exact approaches but only for simpler dynamics [15]. In this paper, we consider both the paths for dynamic security analysis of electric power systems. The first one is based on passivity and barrier certificates; the second one leads to exact safety verification via positive invariance. These two approaches, complement to each other, offer new perspectives and instrumental analytic and numerical tools for security analysis of power systems.

\section{MATHEMATICAL PRELIMINARIES}

In this section, we present some mathematical notions needed for the development of our results.

Definition 1. Consider nonlinear dynamical systems of the form

$$
\dot{x}(t)=f(x(t)), \quad x(0)=x_{0}, \quad t \geq 0,
$$

where $f: \mathbb{R}^{q} \rightarrow \mathbb{R}^{q}$ satisfies the regularity conditions so that the solutions of (1) define a continuous global semiflow on $\mathbb{R}^{q}$. Let $s(t, x)$ denote the solution of (1) at time $t$ with the initial condition $x$. Given a lower semi-continuous function $V: \mathbb{R}^{q} \rightarrow \mathbb{R}$, the upper right Dini derivative of $V$ along the solution of (1) is defined by $\dot{V}(s(t, x))=\limsup _{h \rightarrow 0^{+}} \frac{V(s(t+h, x))-V(s(t, x))}{h}$.

Definition 2 ([16]). Let $V: \mathbb{R}^{q} \rightarrow \mathbb{R}$ be a locally Lipschitz continuous function. The Clarke upper generalized derivative of $V(\cdot)$ at $x$ in the direction of $v \in \mathbb{R}^{q}$ is defined by $V^{o}(x, v) \triangleq$ $\limsup _{y \rightarrow x, h \rightarrow 0^{+}} \frac{V(y+h v)-V(y)}{h}$. The Clarke generalized gradient $\partial V: \mathbb{R}^{q} \rightarrow \mathcal{B}\left(\mathbb{R}^{q}\right)$ of $V(\cdot)$ at $x$ is the set

$$
\partial V(x) \triangleq \operatorname{co}\left\{\lim _{i \rightarrow \infty} \nabla V\left(x_{i}\right): x_{i} \rightarrow x, x_{i} \notin \mathcal{N} \cup \mathcal{S}\right\}
$$

where "co" denotes the convex hull, $\nabla$ denotes the nabla operator, $\mathcal{N}$ is the set of measure zero where $\nabla V$ does not exist, and $S$ is an arbitrary set of measure zero in $\mathbb{R}^{q}$.

Note that $V^{o}(x, v)$ always exists. Furthermore (2) is well defined and consists of all convex combinations of all the possible limits of the gradient at neighboring points where $V$ is differentiable. In order to state the main results of this paper, we need some additional notation and definitions. Given a locally Lipschitz continuous function $V: \mathbb{R}^{q} \rightarrow \mathbb{R}$, the set-valued Lie derivative $\mathcal{L}_{f} V: \mathbb{R}^{q} \rightarrow \mathcal{B}(\mathbb{R})$ of $V$ with respect to $f$ at $x[16]$ is defined as $\mathcal{L}_{f} V(x) \triangleq\{a \in \mathbb{R}:$ there exists $v \in \mathcal{K}[f](x)$ such that $p^{\mathrm{T}} v=a$ for all $\left.p \in \partial V(x)\right\}$, where $\mathcal{K}[f](x)$ is defined in (11). If $V(\cdot)$ is continuously differentiable at $x$, then $\mathcal{L}_{f} V(x)=\{\nabla V(x)$. $v, v \in \mathcal{K}[f](x)\}$. In the case where $\mathcal{L}_{f} V(x)$ is nonempty, we use the notation $\max \mathcal{L}_{f} V(x)$ to denote the largest element of $\mathcal{L}_{f} V(x)$.

\section{POWER NETWORK SYSTEM MODEL}

Consider an $n$-machine network-reduction power system given by the three dimensional flux decay model [5]

$$
\begin{aligned}
\dot{\delta}_{i} & =\omega_{i 0} \omega_{M i}, \\
M_{i} \dot{\omega}_{M i} & =-D_{M i} \omega_{M i}+P_{m i}-V_{q i} \\
\times & \sum_{j=1, j \neq i}^{n} V_{q j}\left[G_{M i j} \cos \left(\delta_{i}-\delta_{j}\right)+B_{M i j} \sin \left(\delta_{i}-\delta_{j}\right)\right], \\
T_{d i} \dot{V}_{q i} & =-\left[1-B_{M i i}\left(x_{d i}-x_{d i}^{\prime}\right)\right] V_{q i}-\left(x_{d i}-x_{d i}^{\prime}\right) \\
\times & \sum_{j=1, j \neq i}^{n} V_{q j}\left[G_{M i j} \sin \left(\delta_{i}-\delta_{j}\right)-B_{M i j} \cos \left(\delta_{i}-\delta_{j}\right)\right] \\
& +E_{f s i}+u_{f i}, \quad i=1,2, \ldots, n,
\end{aligned}
$$

where $\delta_{i}$ represents the rotor angle, $\omega_{M i}$ represents the rotor speed, $V_{q i}$ represents the quadrature axis internal voltage. Furthermore, the control input is the field excitation signal $u_{f i}$. The 
parameters $G_{M i j}=G_{M j i}$ and $B_{M i j}=B_{M j i}$ are, respectively, the conductance and susceptance of the generator $i$. $E_{f s i}$ represents the constant component of the field voltage and $P_{m i}$ the mechanical power, which is assumed to be a constant. The parameters $x_{d i}, x_{d i}^{\prime}, \omega_{i 0}$, and $D_{M i}$ represent the direct axis synchronous reactance, the direct axis transient reactance, the synchronous speed, and damping coefficient, respectively. Note that all the parameters are positive and $x_{d i}>x_{d i}^{\prime}$.

To simplify the model, we introduce the parameters $k_{i} \triangleq$ $E_{f s i} / T_{d i}, a_{i} \triangleq D_{M i} / M_{i}, c_{i} \triangleq\left(P_{m i} \omega_{i 0}\right) / M_{i}, d_{i j} \triangleq\left(G_{M i j} \omega_{i 0}\right) / M_{i}$, $b_{i j} \triangleq\left(B_{M i j} \omega_{i 0}\right) / M_{i}, Z_{i j} \triangleq \sqrt{d_{i j}^{2}+b_{i j}^{2}}, \alpha_{i j} \triangleq \arctan \left(d_{i j} / b_{i j}\right), h_{i} \triangleq$ $\left[1-B_{M i j}\left(x_{d i}-x_{d i}^{\prime}\right)\right]$, and $r_{i} \triangleq\left(x_{d i}-x_{d i}^{\prime}\right) / T_{d i}$. Furthermore, define the state variables as $x_{1 i} \triangleq \delta_{i}, x_{2 i} \triangleq \omega_{i}$, and $x_{3 i} \triangleq V_{q i}$, and the control input as $u_{i} \triangleq u_{f i} / T_{d i}$. Then (3-5) can be rewritten as

$$
\begin{aligned}
\dot{x}_{1 i}(t) & =x_{2 i}(t), \\
\dot{x}_{2 i}(t) & =-a_{i} x_{2 i}(t)+c_{i}-x_{3 i}(t) \\
& \times \sum_{j=1, j \neq i}^{n} x_{3 j}(t) Z_{i j} \sin \left(x_{1 i}(t)-x_{1 j}(t)+\alpha_{i j}\right), \\
\dot{x}_{3 i}(t) & =-h_{i} x_{3 i}(t)+k_{i}+u_{i}(t)+r_{i} \\
& \times \sum_{j=1, j \neq i}^{n} x_{3 j}(t) Z_{i j} \cos \left(x_{1 i}(t)-x_{1 j}(t)+\alpha_{i j}\right),
\end{aligned}
$$

where $i=1, \ldots, n$. Note that if $d_{i j}=0$, then $\alpha_{i j}=0$.

\section{SAFETY VERIFICATION: PASSIVITY-BASED AP- PROACH}

In this section, we consider an approximate safety verification method using barrier certificates [14] and extend it to discontinuous dynamics. Passivity structure and energy shaping technique are exploited to obtain new safety conditions.

\subsection{Safety Verification using Barrier Certificates}

Consider a nonlinear dynamical system $\mathcal{G}$ given by

$$
\dot{x}(t)=f(x(t), u(t)), \quad x(0)=x_{0}, \quad t \geq 0,
$$

where $x(t) \in \mathbb{R}^{n}, u(t) \in \mathcal{U} \subseteq \mathbb{R}^{m}$, and $f(t) \triangleq f(x(t), u(t))$ is Lebesgue measurable and $f: \mathbb{R}^{n} \times \mathbb{R}^{m}$ is locally essentially bounded [17].

Definition 3 ([14]). Consider $\mathcal{G}$ and assume that $f$ is continuous in $x$ and $u$. Given the state set $\mathcal{S} \subseteq \mathbb{R}^{n}$, the initial set $\mathcal{S}_{0} \subseteq \mathcal{S}$, the unsafe set $\mathcal{S}_{u} \subseteq \mathcal{S}$, and the control input set $\mathcal{U} \subseteq \mathbb{R}^{m}$, we say that the safety property holds if there exist no time instant $T \geq 0$ and a piecewise continuous and bounded control input $u:[0, T] \rightarrow \mathcal{U}$ that gives rise to an unsafe system trajectory, that is, a trajectory $x:[0, T] \rightarrow \mathbb{R}^{n}$ satisfying $x(0) \in \mathcal{S}_{0}, x(T) \in \mathcal{S}_{u}$, and $x(t) \in \mathcal{S}$ for all $t \in[0, T]$.
The following definition is a generalization of Definition 3 from continuous-time dynamical systems to discontinuous $d y$ namical systems, which arise in many electric power systems [18]. More importantly, risk assessment in power industry is particularly interested in the case where the power system topology changes when a fault occurs in power networks. In this case, the power system undergoes switching due to topological changes and this fault is hard to detect a priori. Hence, it is better to use discontinuous dynamical systems to describe the power system under dynamically changing environment than hybrid dynamical systems. Particularly the results in [14] are hard to be implemented on power systems with switching topology.

Definition 4. Consider $\mathcal{G}$ and assume that $f$ is upper semicontinuous. Given the state set $\mathcal{S} \subseteq \mathbb{R}^{n}$, the initial set $\mathcal{S}_{0} \subseteq \mathcal{S}$, the unsafe set $\mathcal{S}_{u} \subseteq \mathcal{S}$, and the control input set $\mathcal{U} \subseteq \mathbb{R}^{m}$, we say that the safety property holds if there exist no time instant $T \geq 0$ and a piecewise continuous and bounded control input $u:[0, T] \rightarrow \mathcal{U}$ that gives rise to an unsafe Filippov solution, that is, a Filippov solution $\psi:[0, T] \rightarrow \mathbb{R}^{n}$ satisfying $\psi(0) \in \mathcal{S}_{0}, \psi(T) \in \mathcal{S}_{u}$, and $\psi(t) \in \mathcal{S}$ for all $t \in[0, T]$.

Note that an absolutely continuous function $x:[0, \tau] \rightarrow \mathbb{R}^{n}$ is said to be a Filippov solution [17] of (9) on the interval [0, $\tau]$ with initial condition $x(0)=x_{0}$, if for given $u(t) \in \mathcal{U}, x(t)$ satisfies

$$
\dot{x}(t) \in \mathcal{K}[f](x(t), u(t)), \quad \text { a.e. } t \in[0, \tau],
$$

where the Filippov set-valued map $\mathcal{K}[f]: \mathbb{R}^{n} \times \mathbb{R}^{m} \rightarrow \mathcal{B}\left(\mathbb{R}^{n}\right)$ is defined by

$$
\mathcal{K}[f](x, u) \triangleq \bigcap_{\delta>0} \bigcap_{\mu(\mathcal{S})=0} \overline{\operatorname{co}}\left\{f\left(\mathcal{B}_{\delta}(x) \backslash \mathcal{S}, u\right)\right\}, x \in \mathbb{R}^{n},
$$

where $\mathcal{B}\left(\mathbb{R}^{n}\right)$ denotes the collection of all subsets of $\mathbb{R}^{n}, \mu(\cdot)$ denotes the Lebesgue measure in $\mathbb{R}^{n}$, and "co" denotes the convex closure. Note that $\mathcal{K}[f]: \mathbb{R}^{n} \rightarrow \mathcal{B}\left(\mathbb{R}^{n}\right)$ is a map that assigns sets to points. Dynamical systems of the form given by (10) are called differential inclusions and for each state $x \in \mathbb{R}^{n}$, they specify a set of possible evolutions rather than a single one.

Definitions 3 and 4 are very general for $\mathcal{G}$. But it is difficult to verify them for a given system since it requires the information of system trajectories. To overcome this, [14] proposed a Lyapunov-like approach to verify safety for nonlinear systems using barrier certificates. The continuum version of safety verification using barrier certificates can be found in [19]. Next, we generalize these Lyapunov-like results to discontinuous dynamical systems and discontinuous barriers. First, we consider the case where (9) is a discontinuous dynamical system.

Theorem 1. Consider a discontinuous dynamical system (9) with $f$ being upper semi-continuous. Suppose there exists a lo- 
cally Lipschitz continuous function $B: \mathbb{R}^{n} \rightarrow \mathbb{R}$ such that

$$
\begin{aligned}
B(x) & \leq 0, x \in \mathcal{S}_{0}, \\
B(x) & >0, x \in \mathcal{S}_{u}, \\
\max \mathcal{L}_{f} B(x) & \leq 0 \text { or } \mathcal{L}_{f} B(x)=\varnothing, \text { a.e. }(x, u) \in \mathcal{S} \times \mathcal{U},
\end{aligned}
$$

then the safety of the system in the sense of Definition 4 is guaranteed. Alternatively, if all the above conditions are satisfied except (14) is replaced by $\max \mathcal{L}_{f} B(x)<0$ or $\mathcal{L}_{f} B(x)=\varnothing$ a.e. $(x, u) \in \mathcal{S} \times \mathcal{U}$ such that $B(x)=0$, then the safety of the system in the sense of Definition 4 is guaranteed.

Proof. Conversely, assume that there exists a barrier certificate $B(x)$ satisfying (12)-(14) and at the same time the system is not safe, that is, there exist a time instant $T \geq 0$, a control input $u:[0, T] \rightarrow \mathcal{U}$, and an initial condition $x_{0} \in \mathcal{S}_{0}$ such that a Filippov solution $x(t)$ of the system starting at $x(0)=x_{0}$ satisfies $x(t) \in \mathcal{S}$ for all $t \in[0, T]$ and $x(T) \in \mathcal{S}_{u}$. Since either $\max \mathcal{L}_{f} B(x) \leq 0$ or $\mathcal{L}_{f} B(x)=\varnothing$ for almost every $(x, u) \in \mathcal{S} \times \mathcal{U}$, it follows from Lemma 1 of [16] that $\frac{\mathrm{d}}{\mathrm{d} t} B(x(t))$ exists and is contained in $\mathcal{L}_{f} B(x(t))$ for almost every $t \geq 0$. Now, by assumption, $B(x(t))-B(x(\tau))=\int_{\tau}^{t} \frac{\mathrm{d}}{\mathrm{ds}} B(x(s)) \mathrm{d} s \leq 0, t \geq \tau$. In particular, $B(x(T)) \leq B(x(0))$. This contradicts (12) and (13). Hence, the system must be safe.

For the second conclusion, suppose that a control input $u:[0, T] \rightarrow \mathcal{U}$ and a corresponding unsafe Filippov solution $x:[0, T] \rightarrow \mathcal{S}$ exist. Let $t_{1}$ and $t_{2}$ be two time instants such that $0 \leq t_{1}<t_{2} \leq T, B\left(x\left(t_{1}\right)\right) \leq 0, B\left(x\left(t_{2}\right)\right) \geq 0$, and $\frac{\mathrm{d}}{\mathrm{d} t} B(x(t))<0$ for almost all $t \in\left[t_{1}, t_{2}\right]$. Then the assertion follows using the similar arguments as above.

A function $B(x)$ satisfying (12) and (13) is called a barrier. Theorem 1 considers the case where $B(x)$ is a continuous barrier. Next, we extend Theorem 1 to the case where $B(x)$ is a discontinuous barrier.

Theorem 2. Consider a nonlinear dynamical system (9) with $f$ being Lipschitz continuous. Suppose there exists a lower semi-continuous function $B: \mathbb{R}^{n} \rightarrow \mathbb{R}$ such that (i) $B(x) \leq$ $0, \forall x \in \mathcal{S}_{0}$; (ii) $B(x)>0, \forall x \in \mathcal{S}_{u}$; and (iii) $\dot{B}(x(t)) \leq 0, \forall t \geq$ $0, \forall\left(x_{0}, u(t)\right) \in \mathcal{S}_{0} \times \mathcal{U}$, then the safety of the system in the sense of Definition 3 is guaranteed.

Proof. The proof is similar to the proof of Theorem 1 and hence, is omitted.

It follows from Theorems 1 and 2 that verifying safety for $G$ reduces to finding a Lyapunov-like function $B(x)$. However, finding an appropriate Lyapunov-like function is notoriously difficult. For the vector field being polynomials, an approximation method based on sums-of-squares optimization is presented in [14]. This method is quite restrictive since the vector fields of many electric power systems are not polynomials.

\subsection{Safety Verification using Passivity}

In this subsection, we propose a passivity-based approach to verify safety of power systems by taking advantage of the structure of power systems. Specifically, since many electrical systems such as power systems possess power-conserving structure, we may formulate the power system as a port-Hamiltonian system [20]. Using dissipative system theory [21], it can be shown that this port-Hamiltonian system satisfies a passive condition, which yields a much simpler safety condition that can be checked analytically without much computation. This gives an efficient way to do risk assessment for power systems compared with some traditional method in [9].

To start with our discussion, we put the power network model (6)-(8) in the following port-Hamiltonian form

$$
\begin{aligned}
\dot{x} & =[\mathcal{I}(x)-\mathcal{R}(x)] \nabla^{\mathrm{T}} \mathcal{H}(x)+G(x) u, \\
y & =G^{\mathrm{T}}(x) \nabla^{\mathrm{T}} \mathcal{H}(x) .
\end{aligned}
$$

Let $S \subseteq \mathbb{R}^{n}$ be a given open set.

Lemma 1. Suppose $\mathcal{H}$ is locally Lipschitz continuous. Furthermore, assume (i) $\mathcal{H}(x) \leq a, \forall x \in \mathcal{S}_{0}$; (ii) $\mathcal{H}(x)>b, \forall x \in \mathcal{S}_{u}$; and

$$
u^{\mathrm{T}} y \leq \nabla \mathcal{H}(x) \mathcal{R}(x) \nabla^{\mathrm{T}} \mathcal{H}(x),(x, u) \in \mathcal{S} \times \mathcal{U},
$$

where $a, b \in \mathbb{R}$ and $a \leq b$. Then the safety of the system in the sense of Definition 3 is guaranteed.

Note that if $a=b=0$, then $\mathcal{H}$ in Lemma 1 becomes a barrier. Also, Lemma 1 implies that if the level set of the Hamiltonian function happens to be a barrier, then the power system using passivity-based control is always safe. However, in general $\mathcal{H}$ need not be a barrier. In this case, security analysis becomes much more involved. Here, we first consider the simple case where the barriers are contained in the level set of the Hamiltonian function.

Theorem 3. Suppose $\mathcal{H}$ is locally Lipschitz continuous and the barrier certificate $B(x)$ of $(15)$ and (16) is given, that is, $B(x) \leq$ $0, \forall x \in \mathcal{S}_{0}$ and $B(x)>0, \forall x \in \mathcal{S}_{u}$ Assume $B^{-1}((-\infty, 0]) \subseteq$ $\mathcal{H}^{-1}((-\infty, a])$ and $B^{-1}([0,+\infty)) \subseteq \mathcal{H}^{-1}([b,+\infty))$, where $a \leq b$. Furthermore, assume (17) holds. Then the power system given by (15) and (16) is safe in the sense of Definition 3. Alternatively, if (17) holds almost everywhere, then the power system given by (15) and (16) is safe in the sense of Definition 4.

Proof. Note that $\mathcal{H}(x) \leq a, x \in \mathcal{S}_{0}, \mathcal{H}(x)>b, x \in \mathcal{S}_{u}$, and $\mathcal{L}_{f} \mathcal{H}(x) \leq 0, x \in \mathcal{S}$. The assertion now follows from Lemma 1.

The following corollary says that if the unsafe set happens to be the set of all minimum points of the Hamiltonian function, then the power system using passivity-based control is always safe. In this case, the barrier is always the minimum level set of the Hamiltonian function. 
Corollary 1. Assume $\mathcal{H}$ is locally Lipschitz continuous and bounded below. Furthermore, assume (i) $\mathcal{H}(x)=$ $\inf _{z \in \mathcal{S}} \mathcal{H}(z), \forall x \in \mathcal{S}_{0}$; (ii) $\mathcal{H}(x)>\inf _{z \in \mathcal{S}} \mathcal{H}(z), \forall x \in \mathcal{S}_{u}$; and (iii) (17) holds. Then the safety of the system in the sense of Definition 3 is guaranteed.

Remark 1. Motivated by the converse Lyapunov theorems, it is interesting to ask whether the converse (or partial converse) of Theorems 1-3 hold. Note that the converse is always true if a barrier certificate function takes the extended values (e.g., $+\infty)$. For instance, let $B$ be an indicator function defined by $B(x)=0$ if $x \notin \mathcal{S}_{u}$ and $B(x)=+\infty$ if $x \in \mathcal{S}_{u}$. Assuming that $\mathcal{S} \backslash \mathcal{S}_{u}$ is closed, then $B$ is lower semicontinuous and the converse of Theorem 2 holds. However, due to space limitation, we do not further explore this issue here.

\subsection{Safety Verification via Energy Shaping}

Previously, we assume that the barriers are always contained in the level set of the Hamiltonian function. However, this assumption is restrictive since the shape of the barriers could be arbitrarily depicted. Next, we propose an energy shaping-based approach to detect safety issues for power systems. The basic idea is to transform the original Hamiltonian power system into another Hamiltonian system in light of the prescribed barrier. Then the security analysis of the original system becomes the security of the transformed system. This transformation can be achieved via the energy shaping technique [22], which is a powerful control design technique for Hamiltonian systems.

To begin with our approach, we review the energy-shaping method for port Hamiltonian systems. Consider the port Hamiltonian system $\Sigma$ given by (15) and (16). Next, we consider the interconnection of $\Sigma$ with another port Hamiltonian system $\Sigma_{C}$ given by $\dot{x}_{\mathrm{c}}=\left[\mathcal{J}_{\mathrm{c}}\left(x_{\mathrm{c}}\right)-\mathcal{R}_{\mathrm{c}}\left(x_{\mathrm{c}}\right)\right] \nabla^{\mathrm{T}} \mathcal{H}_{\mathrm{c}}\left(x_{\mathrm{c}}\right)+G_{\mathrm{c}}\left(x_{\mathrm{c}}\right) u_{\mathrm{c}}$ and $y_{\mathrm{c}}=G_{\mathrm{c}}^{\mathrm{T}}\left(x_{\mathrm{c}}\right) \nabla^{\mathrm{T}} \mathcal{H}_{\mathrm{c}}\left(x_{\mathrm{c}}\right)$ regarded as the controller via the standard feedback interconnection $u=-y_{\mathrm{c}}+e$ and $u_{\mathrm{c}}=y+e_{\mathrm{c}}$, with $e$ and $e_{\mathrm{c}}$ external signals inserted in the feedback loop and $x_{\mathrm{c}}=\left[x_{\mathrm{c} 1}, \ldots, x_{\mathrm{c} n_{\mathrm{c}}}\right] \in \mathbb{R}^{n_{\mathrm{c}}}$. Then by using the Energy-Casimir method [22], we obtain the following result.

Lemma 2 ([22]). Consider the feedback interconnected port Hamiltonian system given by $\Sigma$ and $\Sigma_{C}$ for $e_{\mathrm{c}}=0$ and $n=n_{\mathrm{c}}$. Let $g=\left[g_{1}, \ldots, g_{n_{\mathrm{c}}}\right]$ satisfy

$$
\begin{aligned}
\nabla g(x) \mathcal{I}(x) \nabla^{\mathrm{T}} g(x) & =\mathcal{I}_{\mathrm{c}}\left(x_{\mathrm{c}}\right), \\
\mathcal{R}(x) \nabla^{\mathrm{T}} g(x) & =0, \\
\mathcal{R}_{\mathrm{c}}\left(x_{\mathrm{c}}\right) & =0, \\
\nabla g(x) \mathcal{I}(x) & =G_{\mathrm{c}}\left(x_{\mathrm{c}}\right) G^{\mathrm{T}}(x) .
\end{aligned}
$$

Furthermore, assume that $g_{\mathrm{c}}\left(x_{\mathrm{c}}\right)=0$ and $G_{\mathrm{c}}\left(x_{\mathrm{c}}\right)$ is injective. Then the reduced dynamics on any multi-level set $L_{C}=\left\{\left(x, x_{\mathrm{c}}\right)\right.$ :

$$
\begin{aligned}
x_{\mathrm{c} i}=g_{i}(x)+c_{i}, i & \left.=1, \ldots, n_{\mathrm{c}}\right\} \\
\dot{x} & =[\mathcal{I}(x)-\mathcal{R}(x)] \nabla^{\mathrm{T}} \mathcal{H}_{\mathrm{s}}(x)+G(x) e, \\
y & =G^{\mathrm{T}}(x) \nabla^{\mathrm{T}} \mathcal{H}_{\mathrm{s}}(x),
\end{aligned}
$$

where $\mathcal{H}_{\mathrm{s}}(x) \triangleq \mathcal{H}(x)+\mathcal{H}_{\mathrm{c}}(g(x)+c), c=\left[c_{1}, \ldots, c_{n_{\mathrm{c}}}\right]^{\mathrm{T}}$.

We call $\mathcal{H}_{\mathcal{S}}(x)$ an energy shaped Hamiltonian. There are many efforts towards solving partial differential equations (18)(21) to obtain energy shaped Hamiltonian systems. The readers should refer to [23] for the details of solving (18)-(21), [24] for a survey of this method, and [25] for its application to stabilization of power systems. Here we do not focus on how to derive energy shaped Hamiltonian systems. We rather see how to use this method to solve safety verification of power systems. To this point, we assume that we already obtain the desired energy shaped Hamiltonian.

Theorem 4. Suppose the barrier certificate $B(x)$ of (15) and (16) is given, that is, $B(x) \leq 0, x \in \mathcal{S}_{0}$ and $B(x)>0, x \in \mathcal{S}_{u}$. Let $\mathcal{H}_{\mathrm{s}}$ be an energy shaped Hamiltonian. Assume $B^{-1}((-\infty, 0]) \subseteq$ $\mathcal{H}_{\mathrm{s}}^{-1}((-\infty, a])$ and $B^{-1}([0,+\infty)) \subseteq \mathcal{H}_{\mathrm{s}}^{-1}([b,+\infty))$, where $a \leq \bar{b}$. Consider the energy shaped port-controlled Hamiltonian system given by (22) and (23) and the new control input set $\mathcal{U}_{\mathrm{e}}$. If

$$
e^{\mathrm{T}} y \leq \nabla \mathcal{H}_{\mathrm{s}}(x) \mathcal{R}(x) \nabla^{\mathrm{T}} \mathcal{H}_{\mathrm{s}}(x),(x, u) \in \mathcal{S} \times \mathcal{U}_{\mathrm{e}},
$$

then the power system given by (15) and (16) is safe in the sense of Definition 3. Alternatively, if (24) holds almost everywhere, then the power system given by (15) and (16) is safe in the sense of Definition 4.

Proof. Suppose we obtain $\mathcal{H}_{\mathrm{s}}$ via Lemma 2. Then the proof is similar to that of Theorem 3.

\subsection{Applications to Power Systems}

To illustrate the safety verification approach developed in Section 4 , we convert the power system given by (6)-(8) into a port-controlled Hamiltonian system. Specifically, let

$$
\begin{aligned}
\mathcal{H}(x) & \triangleq \sum_{i=1}^{n}\left[\frac{r_{i}}{2 h_{i}} x_{2 i}^{2}-\frac{r_{i} c_{i}}{h_{i}} x_{1 i}-\frac{k_{i}}{h_{i}} x_{3 i}+\frac{1}{2} x_{3 i}^{2}\right. \\
& \left.-\frac{r_{i}}{h_{i}} x_{3 i} \sum_{j=1, j \neq i}^{n} Z_{i j} x_{3 j} \cos \left(x_{1 i}-x_{1 j}+\alpha_{i j}\right)\right],
\end{aligned}
$$

where $\quad x_{i}=\left[x_{1 i}, x_{2 i}, x_{3 i}\right]^{\mathrm{T}}, \quad x=\left[x_{1}^{\mathrm{T}}, \ldots, x_{n}^{\mathrm{T}}\right]^{\mathrm{T}}, \quad$ and $i=1, \ldots, n$. Then (6)-(8) can be rewritten as $\dot{x}_{i}=$ $\left[g_{i}\left(x_{i}\right)-\mathcal{R}_{i}\left(x_{i}\right)\right]\left(\frac{\partial \mathcal{H}(x)}{\partial x_{i}}\right)^{\mathrm{T}}+G_{i}\left(x_{i}\right) u_{i}$, where $G_{i}\left(x_{i}\right)=\left[\begin{array}{lll}0 & 0 & 1\end{array}\right]^{\mathrm{T}}$, $g_{i}\left(x_{i}\right)=\left[\begin{array}{ccc}0 & \frac{h_{i}}{r_{i}} & 0 \\ -\frac{h_{i}}{r_{i}} & 0 & 0 \\ 0 & 0 & 0\end{array}\right], \mathcal{R}_{i}\left(x_{i}\right)=-\left[\begin{array}{ccc}0 & 0 & 0 \\ 0 & \frac{a_{i} h_{i}}{r_{i}} & 0 \\ 0 & 0 & h_{i}\end{array}\right]$ Here, we 
define the system output $y_{i}$ as $y_{i}=G_{i}^{\mathrm{T}}\left(x_{i}\right)\left(\frac{\partial \mathcal{H}(x)}{\partial x_{i}}\right)^{\mathrm{T}}$. Let $B(x)$ be a given barrier certificate for this power system. If (I): $B^{-1}((-\infty, 0]) \subseteq \mathcal{H}^{-1}((-\infty, a])$ and $B^{-1}([0,+\infty)) \subseteq \mathcal{H}^{-1}([b,+\infty))$, where $a \leq b$, and (II): $\sum_{i=1}^{n} u_{i} y_{i} \leq \sum_{i=1}^{n} \frac{\partial \mathcal{H}(x)}{\partial x_{i}} \mathcal{R}_{i}\left(x_{i}\right)\left(\frac{\partial \mathcal{H}(x)}{\partial x_{i}}\right)^{\mathrm{T}}$, then it follows from (3) that the power system given by (6)-(8) is safe in the sense of Definition 3. Alternatively, if the condition (II) holds almost everywhere except for some sets of measure zero in the state space, then (6)-(8) is safe in the sense of Definition 4. Finally, if the barrier certificate $B(x)$ is not contained in the level set of $\mathcal{H}(x)$, then one can try energy shaping so that the conditions in Theorem 4 are satisfied. If this is the case, then the sufficient condition for guaranteeing safety is given by Theorem 4 . Otherwise, the method is invalid and a new investigation is needed for solving the safety verification problem. Due to space limitation, we do not discuss this case here.

\section{SAFETY VERIFICATION: POSITIVE INVARIANCE APPROACH}

Linear models are popular in power system analysis and control because of its simplicity and good approximation. When the operating condition of the power system is close to a nominal equilibrium, the linear model is sufficiently accurate to describe the dynamic behavior of the power system. This model also reduces complexity of security analysis by using well-developed linear system theory. In this section, we utilize linear models of the power system along with special structure of constraint regions to address exact security verification of power systems.

\subsection{Exact Safety Verification using Positive Invari- ance}

Roughly speaking, a set is positively invariant with respect to a dynamical system if a system trajectory starting from the given set will remain in that set for all positive times. The concept of positive invariance is essential in asymptotic analysis of dynamical systems and Lyapunov stability theory. It has also been lately recognized that positive invariance of a hybrid system plays a crucial role in characterization of a variety of important long-time hybrid dynamic behavior.

The positive invariance technique developed in [26] has been exploited to address analytical and computational issues of safety verification of affine dynamics on semialgebraic sets. This method makes use of the affine dynamic models widely used in power system analysis and control and the algebraic structure of constraint regions. This allows us to obtain less conservative and computationally tractable verification results.

Suppose the linear power network model is given by $\mathbf{S}: \dot{x}=$ $A x+d$, where $x \in \mathbb{R}^{n}, A \in \mathbb{R}^{n \times n}$, and $d \in \mathbb{R}^{n}$. For the given $A$ and $d$, we can uniquely decompose $d$ into $d=d_{c}+d_{n}$, where $d_{c}$ is the orthogonal projection of $d$ onto the column space of $A$ and $d_{n}$ is the projection onto the null space of $A^{\mathrm{T}}$ such that $d_{c}$ is orthogonal to $d_{n}$. Since $d_{c}=A u_{c}$ for some vector $u_{c}$, the state transformation $\tilde{x}=x+u_{c}$ converts the original system into $\dot{\tilde{x}}=A \tilde{x}+d_{n}$. Hence, if $d_{n}=0$, then the original affine system can be transformed into the linear dynamics.

Definition 5 ([26]). Consider $\mathcal{G}$ with $u(t) \equiv 0$. Given sets $\mathcal{S}_{0}$ and $\mathcal{S}_{f}$ in $\mathbb{R}^{n}$ such that $\mathcal{S}_{0} \subseteq \mathcal{S}_{f}$. We say that exact safety property holds on a time interval $\Delta$ if the trajectory $s\left(t, x_{0}\right)$ of $\mathcal{G}$ satisfies $s\left(t, x_{0}\right) \in \mathcal{S}_{f}, \forall t \in \Delta, \forall x_{0} \in \mathcal{S}_{0}$.

Next consider the polyhedral initial set $\mathcal{S}_{0}$ and the final safe region $\mathcal{S}_{f}$. Here a polyhedron is the set of the form $\mathcal{P}=\left\{x \in \mathbb{R}^{n}: C x \geq b\right\}$, where $C \in \mathbb{R}^{m \times n}$ and $b \in \mathbb{R}^{m}$. By Minkowski-Weyl Decomposition Theorem, we decompose $\mathcal{S}_{0}$ into the sum of a compact convex hull and a conic hull, i.e., $S_{0}=\operatorname{conv}\left(v^{1}, \ldots, v^{\ell}\right)+\operatorname{cone}\left(u^{1}, \ldots, u^{k}\right)$ for the extreme points $v^{i}$ and the extreme rays $u^{j}$, where conv and cone denote the convex hull and the closed conic hull of the given sets, respectively. Using this decomposition and letting $\mathcal{S}_{f}=\left\{x \in \mathbb{R}^{n}: C x \geq b\right\}$, it follows from Theorem 18 of [26] that the affine dynamics $\mathbf{S}$ is exactly safe from $\mathcal{S}_{0}$ on $\Delta=[0, \infty)$ in the sense of Definition 5 if and only if for all $t \geq 0, C\left[e^{A t} v^{i}+\int_{0}^{t} e^{A(t-\tau)} \mathrm{d} \tau d\right] \geq b$ for all $i=1, \ldots, \ell$ and $C\left[e^{A t} u^{j}+\int_{0}^{t} e^{A(t-\tau)} \mathrm{d} \tau d\right] \geq 0$ for all $j=1, \ldots, k$. These conditions enable one to check for finitely many vectors only and thus considerably simplify computations. We summarize this result as follows.

Proposition 1. Given two polyhedral sets $\mathcal{S}_{0}$ and $\mathcal{S}_{f}=\{x \in$ $\left.\mathbb{R}^{n}: C x \geq b\right\}$. Let $\mathcal{S}_{0}=\operatorname{conv}\left(v^{1}, \ldots, v^{\ell}\right)+\operatorname{cone}\left(u^{1}, \ldots, u^{k}\right)$ for the extreme points $v^{i}$ and the extreme rays $u^{j}$. Then the affine dynamics $\mathbf{S}$ is exactly safe from $\mathcal{S}_{0}$ on $\Delta=[0, \infty)$ in the sense of Definition 5 if and only if for all $t \geq 0, C\left[e^{A t} v^{i}+\int_{0}^{t} e^{A(t-\tau)} \mathrm{d} \tau d\right] \geq$ $b$ for all $i=1, \ldots, \ell$ and $C\left[e^{A t} u^{j}+\int_{0}^{t} e^{A(t-\tau)} \mathrm{d} \tau d\right] \geq 0$ for all $j=$ $1, \ldots, k$.

Analytic results are usually difficult to obtain for general $\mathcal{S}_{0}$ and $S_{f}$ in exact safety verification, and the best way to solve the exact verification problem is to pursue numerical approaches. This yields a critical computability question of whether such the problem is finitely verifiable or decidable. A typical approach to address this issue is to convert the original safety verification problem into a semialgebraic problem and then apply the TarskiSeidenberg decision procedure [27]. Such an approach has been successfully used to prove decidability of several classes of linear dynamics on semialgebraic sets $[15,28]$.

It is shown in [26] that dynamic analysis techniques for positive invariance analysis, along with semialgebraic arguments, lead to improved decidability results. Specifically, consider an affine dynamics whose defining matrix contains complex eigenvalues only. Most of the linear power systems satisfy this condition. Furthermore, assume that $\mathcal{S}_{0}$ and $\mathcal{S}_{f}$ are closed semialgebraic sets, i.e., they are described by finitely many multivariate polynomial equations or inequalities which are neither convex nor polyhedral in general. It is further shown that the exact safety verification problem is decidable even if the ratios of mode frequencies of the dynamics are irrational. 
Proposition 2 ([26]). Let $\mathcal{S}_{0}=\left\{x \in \mathbb{R}^{n}: p(x) \geq 0, w(x)=0\right\}$ and $\mathcal{S}_{f}=\left\{x \in \mathbb{R}^{n}: f(x) \geq 0\right\}$ be closed semialgebraic sets, where $p, w$, and $f$ are vector-valued multivariate polynomials. Suppose that A has only complex eigenvalues which are all known. Then checking exact safety of the affine dynamics $\mathbf{S}$ on the time interval $\Delta=\mathbb{R}$ in the sense of Definition 5 is decidable.

The following example from [26] illustrates how to convert a safety verification problem into a semi-algebraic problem .

Example 1. Consider the linear system on $\mathbb{R}^{8}$ whose defining matrix $A=\operatorname{diag}\left(A_{1}, A_{2}, A_{3}, A_{4}\right)$. Here the matrix blocks $A_{i}$ are $A_{1}=\left[\begin{array}{cc}\sigma_{1} & \omega_{1} \\ -\omega_{1} & \sigma_{1}\end{array}\right], A_{2}=\left[\begin{array}{cc}0 & \pi \\ -\pi & 0\end{array}\right], A_{3}=\left[\begin{array}{cc}0 & 2 \pi \\ -2 \pi & 0\end{array}\right], A_{4}=$ $\left[\begin{array}{cc}0 & 1 \\ -1 & 0\end{array}\right]$, where $\sigma_{1} \neq 0$ and $\omega_{1}>0$. Let the initial set $\mathcal{S}_{0}=\left\{x \in \mathbb{R}^{8} \mid\left\|x-x^{*}\right\|_{2}^{2} \leq 1\right\}$ for a given $x^{*}$ and the safe region $\mathcal{S}_{f}=\left\{x \in \mathbb{R}^{8} \mid c^{T} x \geq b\right\}$ for some $c \in \mathbb{R}^{8}$ and $b \in$ $\mathbb{R}$. To simplify notation, let $c^{T}=\left(c_{1}^{T}, \cdots, c_{4}^{T}\right)$ and $x=$ $\left(\left(x^{1}\right)^{T}, \cdots,\left(x^{4}\right)^{T}\right)^{T}$, where $c_{i}, x^{i} \in \mathbb{R}^{2}$ correspond to the matrix block $A_{i}$, and let the symplectic matrix $S=\left[\begin{array}{cc}0 & -1 \\ 1 & 0\end{array}\right]$. Therefore, $c^{T} e^{A t} x=e^{\sigma_{1} t}\left[c_{1}^{T} x^{1} \cos \left(\omega_{1} t\right)+\left(S c_{1}\right)^{T} x^{1} \sin \left(\omega_{1} t\right)\right]+q_{1}(x, t)+$ $q_{2}(x, t)$, where $q_{1}(t, x)=c_{2}^{T} x^{2} \cos (\pi t)+\left(S c_{2}\right)^{T} x^{2} \sin (\pi t)+$ $c_{3}^{T} x^{3} \cos (2 \pi t)+\left(S c_{3}\right)^{T} x^{3} \sin (2 \pi t)$ and $q_{2}(t, x)=c_{4}^{T} x^{4} \cos (t)+$ $\left(S c_{4}\right)^{T} x^{4} \sin (t)$. Notice that $q_{1}(t, x)$ and $q_{2}(t, x)$ are periodic in $t$ but their frequency ratio is irrational. Moreover, for a fixed $x$, even though $q_{1}$ is the sum of two sinusoidal functions with frequencies $\pi$ and $2 \pi$ respectively, the maximal (resp. minimal) values of $q_{1}$ cannot be simply written as the sum of the maximal (resp. minimal) values of the two sinusoidal functions. This is why we introduce the time derivative of $q_{1}$ to characterize the extremal value of $q_{1}$. Now define $u_{1} \equiv \cos (\pi t), v_{1} \equiv \sin (\pi t)$ and $u_{2} \equiv \cos (t), v_{2} \equiv \sin (t)$. Rewriting $q_{i}(t, x)$ and $\frac{\partial q_{i}(t, x)}{\partial t}, i=1,2$ in terms of $x, u_{1}, v_{1}, u_{2}, v_{2}$ and applying the argument in [26, proposition 29], we transform the safety verification problem on the time domain $\Delta=\mathbb{R}$ into the following semi-algebraic decision problem $[P \Rightarrow Q]$ on $\mathbb{R}^{12}$, where $P \equiv\left[\pi\left(-c_{2}^{T} x^{2} v_{1}+\right.\right.$ $\left.\left.\left(S c_{2}\right)^{T} x^{2} u_{1}-4 c_{3}^{T} x^{3} u_{1} v_{1}+2\left(S c_{3}\right)^{T} x^{3}\left(u_{1}^{2}-v_{1}^{2}\right)\right)=0\right] \wedge\left[u_{1}^{2}+v_{1}^{2}-\right.$ $1=0] \wedge\left[-c_{4}^{T} x^{4} v_{2}+\left(S c_{4}\right)^{T} x^{4} u_{2}=0\right] \wedge\left[u_{2}^{2}+v_{2}^{2}-1=0\right] \wedge$ $\left[\left(x-x^{*}\right)^{T}\left(x-x^{*}\right)-1 \leq 0\right]$ and $Q \equiv\left[c_{2}^{T} x^{2} u_{1}+\left(S c_{2}\right)^{T} x^{2} v_{1}+\right.$ $\left.c_{3}^{T} x^{3}\left(u_{1}^{2}-v_{1}^{2}\right)+2\left(S c_{3}\right)^{T} x^{3} u_{1} v_{1}+c_{4}^{T} x^{4} u_{2}+\left(S c_{4}\right)^{T} x^{4} v_{2}-b \geq 0\right] \wedge$ $\left[c_{1}^{T} x^{1}=0\right] \wedge\left[\left(S c_{1}\right)^{T} x^{1}=0\right]$. The latter problem is decidable and can be solved using the quantifier elimination technique.

\subsection{Applications to Linear Hamiltonian Systems}

By making use of Hamiltonian structure, we show that Proposition 2 can be improved even for a dynamic matrix with certain repeated real eigenvalues. Consider the (uncontrolled) stable linear Hamiltonian system in canonical form: $\dot{x}=A x$, where $A \in \mathbb{R}^{n \times n}$ is skew symmetric. The matrix $A$ has pure imaginary eigenvalues $\pm \iota \omega_{i}, i=1, \cdots, \ell$ with real $\omega_{i}>0$ and $(n-2 \ell)$ zero eigenvalues. More- over, there exists an orthogonal matrix $U \in \mathbb{R}^{n \times n}$ such that $A=U D U^{T}$, where $D=\operatorname{diag}(J_{1}, \cdots, J_{\ell}, \underbrace{0, \cdots, 0}_{(n-2 \ell)-\text { copies }})$ is a block diagonal matrix with the blocks $J_{i}=\left[\begin{array}{cc}0 & \omega_{i} \\ -\omega_{i} & 0\end{array}\right], \quad i=$ $1, \cdots, \ell$ Thus $e^{A t}=U \operatorname{diag}\left(e^{J_{1} t}, \cdots, e^{J_{\ell} t}, 1, \cdots, 1\right) U^{T}$, where $e^{J_{i} t}=\left[\begin{array}{cc}\cos \left(\omega_{i} t\right) & \sin \left(\omega_{i} t\right) \\ -\sin \left(\omega_{i}\right) & \cos \left(\omega_{i} t\right)\end{array}\right], \quad i=1, \cdots, \ell$ Hence, given a multivariate polynomial $q: \mathbb{R}^{n} \rightarrow \mathbb{R}$ and a vector $x \in \mathbb{R}^{n}, q\left(e^{A t} x\right)$ can be expressed as $q\left(e^{A t} x\right)=c(x)+\sum_{j}\left[g_{j}(x) \cos \left(\widetilde{\omega}_{j} t\right)+\right.$ $\left.h_{j}(x) \sin \left(\widetilde{\omega}_{j} t\right)\right]$, where $c, g, h: \mathbb{R}^{n} \rightarrow \mathbb{R}$ are multivariate polynomials, and each $\widetilde{\omega}_{j}$ is a multiple of one of $\omega_{i}, i=1, \cdots, \ell$. Therefore the similar argument in the proof of Proposition 2 [26] gives rise to:

Corollary 2. Let $\mathcal{S}_{0}=\left\{x \in \mathbb{R}^{n}: p(x) \geq 0\right\}$ and $\mathcal{S}_{f}=\left\{x \in \mathbb{R}^{n}\right.$ : $q(x) \geq 0\}$ be closed semialgebraic sets, where $p$ and $q$ are vectorvalued multivariate polynomials. Then exact safety verification of the linear Hamiltonian system on the time intervals $\Delta=\mathbb{R}_{+}$ or $\Delta=\mathbb{R}$ in the sense of Definition 5 is decidable.

The above corollary can be further strengthened if both $\mathcal{S}_{0}$ and $\mathcal{S}_{f}$ are polyhedral, in view of Proposition 1. Let $d=0$, $\mathcal{S}_{0}=\operatorname{conv}\left(v^{1}, \ldots, v^{\ell}\right)+\operatorname{cone}\left(u^{1}, \ldots, u^{k}\right)$, and $\mathcal{S}_{f}=\left\{x \in \mathbb{R}^{n}:\right.$ $C x \geq b\}$. Since $C e^{A t} v^{i}=(C U) e^{D t}\left(U^{T} v^{i}\right)$, each component of $C e^{A t} v^{i}$ can be written as $p(t) \equiv \gamma+\sum_{i=1}^{\ell}\left[\alpha_{i} \cos \left(\omega_{i} t\right)+\beta_{i} \sin \left(\omega_{i} t\right)\right]$, where the constants $\gamma, \alpha_{i}, \beta_{i}$ depend on $C, U, v^{i}$ only. Let $d_{i}(t) \equiv$ $\alpha_{i} \cos \left(\omega_{i} t\right)+\beta_{i} \sin \left(\omega_{i} t\right)$. By considering the rationality of ratios of $\omega_{i}$, we obtain the collection of (disjoint and distinct) equivalent classes $E_{\omega_{j}}=\left\{d_{i}(t) \mid \omega_{i} / \omega_{j}\right.$ is rational $\}$. Each equivalent class $E_{\omega_{j}}$ attains a basis frequency $\widetilde{\omega}_{s}>0$, namely, $\omega_{i} / \widetilde{\omega}_{s}$ is a positive integer for any frequency $\omega_{i}$ associated with the function $d_{i}(t) \in E_{\omega_{j}}$. Let $E_{\widetilde{\omega}_{s}}$ denote the equivalent class and let $q_{\widetilde{\omega}_{s}}(t) \equiv \sum_{d_{i} \in E_{\widetilde{\omega}_{s}}} d_{i}(t)$. Then the following hold: (1) $q_{\widetilde{\omega}_{s}}(\cdot)$ is a real-valued smooth and periodic function with the frequency $\widetilde{\omega}_{s} ;(2)$ if $q_{\widetilde{\omega}_{s}}(\cdot)$ is not identically zero, then it attains the maximal and minimal values $\sigma_{\widetilde{\omega}_{s}}>0$ and $\nu_{\widetilde{\omega}_{s}}<0$ on $(-\infty, \infty)$ respectively; (3) $q_{\widetilde{\omega}_{s}}(\cdot)$ is onto $\left[v_{\widetilde{\omega}_{s}}, \sigma_{\widetilde{\omega}_{s}}\right]$; and (4) the ratio of two basis frequencies associated with two distinct equivalent classes is irrational. Suppose there are $k$ equivalent classes $E_{\widetilde{\omega}_{s}}$ and thus $p(t)=\sum_{s=1}^{k} q_{\widetilde{\omega}_{s}}(t)$. Although each $q_{\widetilde{\omega}_{s}}$ is periodic, $p$ is generally not and hence may not attain its maximum and minimum on $\mathbb{R}_{+}$. Despite this, it is shown in [26, Lemma 5] that $\sup _{[0, \infty)} p(t)=\gamma+\sum_{s=1}^{k} \sigma_{\widetilde{\omega}_{s}}$ and $\inf _{[0, \infty)} p(t)=\gamma+\sum_{s=1}^{k} v_{\widetilde{\omega}_{s}}$. Consequently, $p(t) \geq b_{j}, \forall t \geq 0$ for some entry $b_{j}$ of $b$ if and only if $\gamma+\sum_{s=1}^{k} v_{\widetilde{\omega}_{s}} \geq b_{j}$. Furthermore, the latter inequality is decidable as shown in Proposition 2. The similar technique can be used to treat $C e^{A t} u^{j} \geq 0, \forall t \geq 0$. 
The semialgebraic conditions obtained above can be verified using the quantifier elimination technique. The recent sumof-squares relaxation approach provides a numerically efficient alternative via powerful semidefinite programming techniques. We refer the interested reader to $[14,27,28]$ for additional information.

\section{REFERENCES}

[1] P. Kundur, J. Paserba, V. Ajjarapu, G. Andersson, A. Bose, C. Canizares, N. Hatziargyriou, D. Hill, A. Stankovic, C. Taylor, T. V. Cutsem, and V. Vittal, "Definition and classification of power system stability," IEEE Trans. Power Systems, vol. 19, pp. 1387-1401, 2004.

[2] K. Morison, L. Wang, and P. Kundur, "Power system security assessment," IEEE Power and Energy Magazine, vol. 2, pp. 30-39, 2004.

[3] L. Wang and K. Morison, "Implementation of online security assessment: Tools for reducing the risk of blackouts," IEEE Power and Energy Magazine, vol. 4, pp. 47-59, 2006.

[4] N. Balu, T. Bertram, A. Bose, V. Brandwajn, G. Cauley, D. Curtice, A. Fouad, L. Fink, M. G. Lauby, B. F. Wollenberg, and J. N. Wrubel, "On-line power system security analysis," Proc. of the IEEE, vol. 80, pp. 262-280, 1992.

[5] H.-D. Chiang, C.-C. Chu, and G. Cauley, "Direct stability analysis of electric power systems using energy functions: Theory, applications, and perspective," Proc. of the IEEE, vol. 83, pp. 1497-1529, 1992.

[6] N. A. Tsolas, A. Arapostathis, and P. P. Varaiya, "A structure preserving energy function for power system transient stability analysis," IEEE Trans. Circuits and Systems, vol. CAS-32, pp. 1041-1049, 1985.

[7] M. A. El-Kady and et al, "Dynamic security assessment utilizing the transient energy function method," IEEE Trans. Power Systems, vol. PS-1, pp. 284-291, 1986.

[8] D. J. Hill and I. M. Y. Mareels, "Stability theory for differential/algebraic systems with applications to power systems," IEEE Trans. Circuits and Systems, vol. 37, pp. 14161423, 1990.

[9] C. L. DeMarco and C. A. Canizares, "A vector energy function approach for security analysis of ac/dc systems," IEEE Trans. Power Systems, vol. 7, pp. 1001-1011, 1992.

[10] F. A. Rahimi, M. G. Lauby, J. N. Wrubei, and K. L. Lee, "Evaluation of the transient energy function method for online dynamic security analysis," IEEE Trans. Power Systems, vol. 8, pp. 497-507, 1992.

[11] A. Llamas and et al., "Clarifications of the BCU method for transient stability analysis," IEEE Trans. Power Systems, vol. 10, pp. 210-219, 1995.

[12] P. Kundur, M. Klein, G. J. Rogers, and M. S. Zywno, "Applications of power system stabilizer for enhancement of overall system stability," IEEE Trans. Power Systems, vol. 4, pp. 614-626, 1989.

[13] G. A. Maria, C. Tang, and J. Kim, "Hybrid transient stabil- ity analysis," IEEE Trans. Power Systems, vol. 5, pp. 384393, 1990.

[14] S. Prajna, A. Jadbabaie, and G. J. Pappas, "A framework for worse-case and stochastic safety verification using barrier certificates," IEEE Trans. Autom. Control, vol. 52, pp. 1415-1428, 2007.

[15] G. Lafferriere, G. J. Pappas, and S. Yovine, "Symbolic reachability computation for families of linear vector fields," J. Symbolic Comput., vol. 32, pp. 231-253, 2001.

[16] A. Bacciotti and F. Ceragioli, "Stability and stabilization of discontinuous systems and nonsmooth Lyapunov functions," ESIAM Control Optim. Calculus Variations, vol. 4, pp. 361-376, 1999.

[17] A. F. Filippov, Differential Equations with Discontinuous Right-Hand Sides. Dordrecht, The Netherlands: Kluwer, 1988.

[18] D. Giaouris, S. Banerjee, B. Zahawi, and V. Pickert, "Stability analysis of the continuous-conduction-mode buck converter via Filippov's method," IEEE Trans. Circuit Syst. I: Reg. Papers, vol. 55, pp. 1084-1096, 2008.

[19] S. Prajna and A. Rantzer, "Convex programs for temporal verification of nonlinear dynamical systems," SIAM J. Control Optim., vol. 46, pp. 999-1021, 2007.

[20] B. Maschke, A. van der Schaft, and P. Bredveld, "An intrinsic Hamiltonian formulation of network dynamics: Nonstandard Poisson structures and gyrators," J. Franklin Inst., vol. 329, pp. 923-926, 1992.

[21] J. C. Willems, "Dissipative dynamical systems. Part I: General theory," Arch. Rational Mech. Anal., vol. 45, pp. 321351, 1972.

[22] R. Ortega, A. van der Schaft, B. Maschke, and G. Escobar, "Energy-shaping of port-controlled Hamiltonian systems by interconnection," in Proc. IEEE Conf. Decision Control, Phoenix, AZ, 1999, pp. 1646-1651.

[23] R. Ortega, A. van der Schaft, F. Castanos, and A. Astolfi, "Control by interconnection and standard passivity-based control of port-Hamiltonian systems," IEEE Trans. Autom. Control, vol. 53, pp. 2527-2542, 2008.

[24] R. Ortega and E. Garcia-Canseco, "Interconnection and damping assignment passivity-based control: A survey," Eur. J. Control, vol. 10, pp. 432-450, 2004.

[25] R. Ortega, M. Galaz, A. Astolfi, Y. Sun, and T. Shen, "Transient stabilization of multimachine power systems with nontrivial transfer conductances," IEEE Trans. Autom. Control, vol. 50, pp. 60-75, 2005.

[26] J. Shen, "Positive invariance of constrained affine dynamics and its applications to hybrid systems and safety verification," submitted for publication.

[27] P. Parrilo, "Semidefinite programming relaxations for semialgebraic problems," Mathematical Programming, Ser. B, vol. 96, pp. 293-320, 2003.

[28] H. Yazarel, S. Prajna, and G. J. Pappas, "S.O.S. for safety," in Proc. IEEE Conf. Decision Control, The Paradise Island, Bahamas, 2004, pp. 461-466. 